\title{
Comparing Extracorporeal Knots in Laparoscopy using Knot and Loop Securities
}

\author{
${ }^{1}$ Rasaq Akintunde Akindele, ${ }^{2}$ Adeniyi Olanipekun Fasanu, ${ }^{3}$ Suresh Chandra Mondal \\ ${ }^{4}$ Johnson Olusanmi Komolafe, ${ }^{5}$ Rajneesh Kumar Mishra
}

\begin{abstract}
Background: Laparoscopic knot tying is a basic surgical skill that has been practiced for centuries having their roots in fishing and sailing. ${ }^{1-4}$ The advent of endoscopic surgery placed more challenges on the surgeons and this ever growing skills need to be acquired. Since endoscopic and arthroscopic knots must be delivered over a distance to a tissue with minimal access maintaining tension is more important than the knot configuration chosen. ${ }^{5,6}$ The aim of this article review is to determine which hand tied knot configuration and possibly, suture size, and suture type that would be safe in laparoscopic surgery.
\end{abstract}

Materials and methods: A literature review was performed using PubMed, Springerlink, Highwire press and search engines like Google and Yahoo. The following search terms were used: extracorporeal knot, arthroscopic knots, Roeder's knot, Meltzer's knot, Mishra's knot, Duncan knot, Nicky's knot, SMC knot, Weston knot and Tennessee extracorporeal knot. A total of 48,100 citations were found. Selected papers were screened for further references. Publications that featured illustrations of sliding knots with statistical methods of analysis were selected. More than 20 different sliding knots were used for this review.

Result: Eighty-one articles were reviewed. Most studies have evaluated knot security only and few studies have evaluated simultaneous both loop and knot security and also only a few compared knot and loop securities to the type of suture materials and their sizes. The addition of three RHAPs improves knot security of all sliding knots tested and improves the loop security of most of the sliding knots tested.

Conclusion: The safety of extracorporeal knot depends on knot configuration, especially when further RHAPs are included.

Keywords: Extracorporeal knot, Tissue approximation, Laparoscopic suturing.

How to cite this article: Akindele RA, Fasanu AO, Mondal SC, Komolafe JO, Mishra RK. Comparing Extracorporeal Knots in Laparoscopy using Knot and Loop Securities. World J Lap Surg 2014;7(1):28-32.

Source of support: Nil

\section{Conflict of interest: None}

\footnotetext{
${ }^{1-3}$ Lecturer and Consultant, ${ }^{4}$ Associate Professor, ${ }^{5}$ Professor

1,2,4 Department of Obstetrics and Gynecology, Lautech Teaching Hospital, Osogbo, Osun State, Nigeria

${ }^{3}$ Department of Obstetrics and Gynecology, Malda Medical College and Hospital, Malda, West Bengal, India

${ }^{5}$ Department of Endoscopic Surgery, World Laparoscopy Hospital, Gurgaon, Haryana, India

Corresponding Author: Fasanu Adeniyi Olanipekun, Department of Obstetrics and Gynecology, Lautech Teaching Hospital Osogbo, Osun State, Nigeria, e-mail: pekun2001@yahoo.co.uk
}

\section{INTRODUCTION}

Since 1978 when endoscopic suturing was first used for hemostasis by Semm in his pelviscopic surgery a lot of interest has been stimulated in endoscopic knotting and suturing. ${ }^{1}$ Gastrointestinal intraluminal suturing was not considered a viable technique until 1984, when Buess reported his transanal endoscopic operative procedure for rectal polyps. ${ }^{2}$ At the end of the last century, Roeder described a ligating technique that used a catgut ligature loop with a slipknot for tonsillectomies in children. This technique was modified with a pushrod-application system by Semm and used in pelviscopic surgery. It is now commercially available as the 'Endoloop'. Modification of Roeder's knot, to make it more secure are Meltzer's and Mishra's knot.' Over the years, there has been the development of several extracorporeal knots. A knot should secure tissue approximation, simple, easy, quick and reliable. Any good knot must fulfil two basic qualities: (1) the knot must be properly formed so the suture does not slip or cut into itself, and (2) it must be easily tightened to ensure maximum strength. For a knot to be effective, it must possess the attributes of both knot security and loop security. ${ }^{8,9}$ Knot security is defined as the effectiveness of the knot at resisting slippage when load is applied and depends on three factors: friction, internal interference, and slack between throws. Loop security is the ability to maintain a tight suture loop as a knot is tied..$^{8-11}$ Thus, any tied knot can have good knot security but poor loop security (a loose suture loop), and therefore be ineffective in approximating the tissue edges to be repaired.

\section{MATERIALS AND METHODS}

A literature review was performed using PubMed, Springerlink, Highwire press, and search engines, like Google and Yahoo. The following search terms were used: extracorporeal knot, arthroscopic knots, Roeder's knot, Meltzer's knot, Mishra's knot, Duncan knot, Nicky's knot, SMC knot, Weston knot and Tennessee extracorporeal knot. A total of 48,100 citations were found. Selected papers were screened for further references. Publications that featured illustrations of sliding knots with statistical methods of analysis were selected. More than 20 different sliding knots were used for this review (Table 1). 
Table 1: Different sliding knots in this review

Two half-hitches
Reversed half-hitches
Practical knot (simple version) ${ }^{11-13}$
Practical knot (advanced version) ${ }^{11-13}$
Nicky's knot or taut-line hitch ${ }^{7-14}$
Giant knot ${ }^{15}$
Modified taut line hitch ${ }^{15,16}$
Tennessee slider
Clinch knot ${ }^{14}$
Roeder's knot
Secure knot ${ }^{77}$
Meltzer's knot
Mishra's knot
Duncan loop, blood slipknot, Hangman's knot, easy loop ${ }^{17,18}$
Weston knot ${ }^{18,19}$
SMC knot ${ }^{15}$
Tayside knot
Hangman's knot
Hangman's tie ${ }^{18-22}$

\section{DEFINITIONS}

Post limb: The straight portion of the suture limb purely defined as the suture limb under the most tension.

Wrapping limb: The free portion of the suture limb that wraps around the post limb.

Effective knot: Possesses the attributes of both knot security and loop security.

Knot security: The effectiveness of a knot to resist slippage when load is applied.

Loop security: The ability of a knot to maintain a tight suture loop as a knot is tied. ${ }^{7,17,22}$

\section{COMMONLY USED COMPOUND SLIDING KNOTS}

Compound sliding knots have more than one turn of the wrapping limb around the post (i.e. any sliding knot other than a half hitch). They can be used in situations where the suture slides smoothly and freely through the tissue and anchoring device. They are advantageous since compound sliding knots can be made to slide down the post limb without unraveling or jamming prematurely. Theoretical disadvantages include abrasion of suture against the anchor eyelet, suture cutting through tissue as it slides. ${ }^{3,5,11,13}$ Mishra's knot is one important extracorporeal knot that combines the loop and knot securities of many other extracorporeal knot that is fast gaining wide acceptance by many laparoscopic surgeons. The steps in tying Mishra's knot is highlighted in Figures $1 \mathrm{~A}$ to I.

\section{RESULTS}

Using a servohydraulic materials testing system (MTS model 858, Bionix, Eden Prairie, MN) to test the knot and loop security of each combination of the knots and suture types (ethibond and fiberwire) and using $5 \mathrm{~N}$ preload and critical loop circumference of $30 \mathrm{~mm}$, it was found that in all cases, no knots failed by suture breakage, suggesting that all knots failed by a combination of knot slippage and suture elongation. When tied with no. 2 ethibond suture or no. 2 fiberwire suture, the Weston knot provided the highest load to failure when compared with the other sliding knots. However, the maximum force of the surgeon's knot was significantly higher than the Weston knot when tied with either ethibond or fiberwire suture.

When the sliding knots were tied with three reversing half hitches on alternating posts (RHAPs) using no. 2 ethibond suture, the Weston RHAP, Roeder RHAP, Mishra RHAP, and SMC RHAP provided the highest force to failure. These forces were not significantly different from the force to failure of the surgeon's knot tied with no. 2 ethibond suture.

When the sliding knots were tied with three RHAPs using no. 2 fiberwire suture, the Weston RHAP provided the highest force to failure. This force was not significantly different from the force to failure of the surgeon's knot. In all cases, tying with either no. 2 ethibond or no. 2 fiberwire suture, the addition of 3 RHAPs after a base sliding knot significantly improved the force to failure. Of the sliding knots tied with no. 2 ethibond suture, the Duncan loop, Roeder knot, Weston knot, Mishra knot and Tennessee slider all provided similar loop circumferences at $5 \mathrm{~N}$ of preload, although the loop circumferences associated with these knots were significantly larger than the loop circumference of the surgeon's knot. When tied with no. 2 ethibond suture, the Roeder RHAP, Mishra RHAP, Duncan RHAP and Nicky's RHAP provided the smallest loop circumferences and were not significantly different from the surgeon's knot. Similarly, when tied with no. 2 fiberwire, the Roeder RHAP, Mishra RHAP, Duncan RHAP and Nicky's RHAP provided the smallest loop circumferences and were not significantly different from the surgeon's knot.

Does securing a sliding knot with three RHAPs decrease the loop circumference (improve loop security)? With knots tied with no. 2 ethibond suture, the addition of three RHAPs decreased the loop circumference of the Nicky's knot, Mishra knot, Roeder knot, the SMC knot and the Tennessee slider. No significant difference was found in the Duncan loop or the Weston knot when tied with or without three RHAPs. When tying knots with no. 2 fiberwire, the addition of three RHAPs decreased the loop circumference of the Nicky's knot, the Mishra knot and the Roeder knot. No significant 

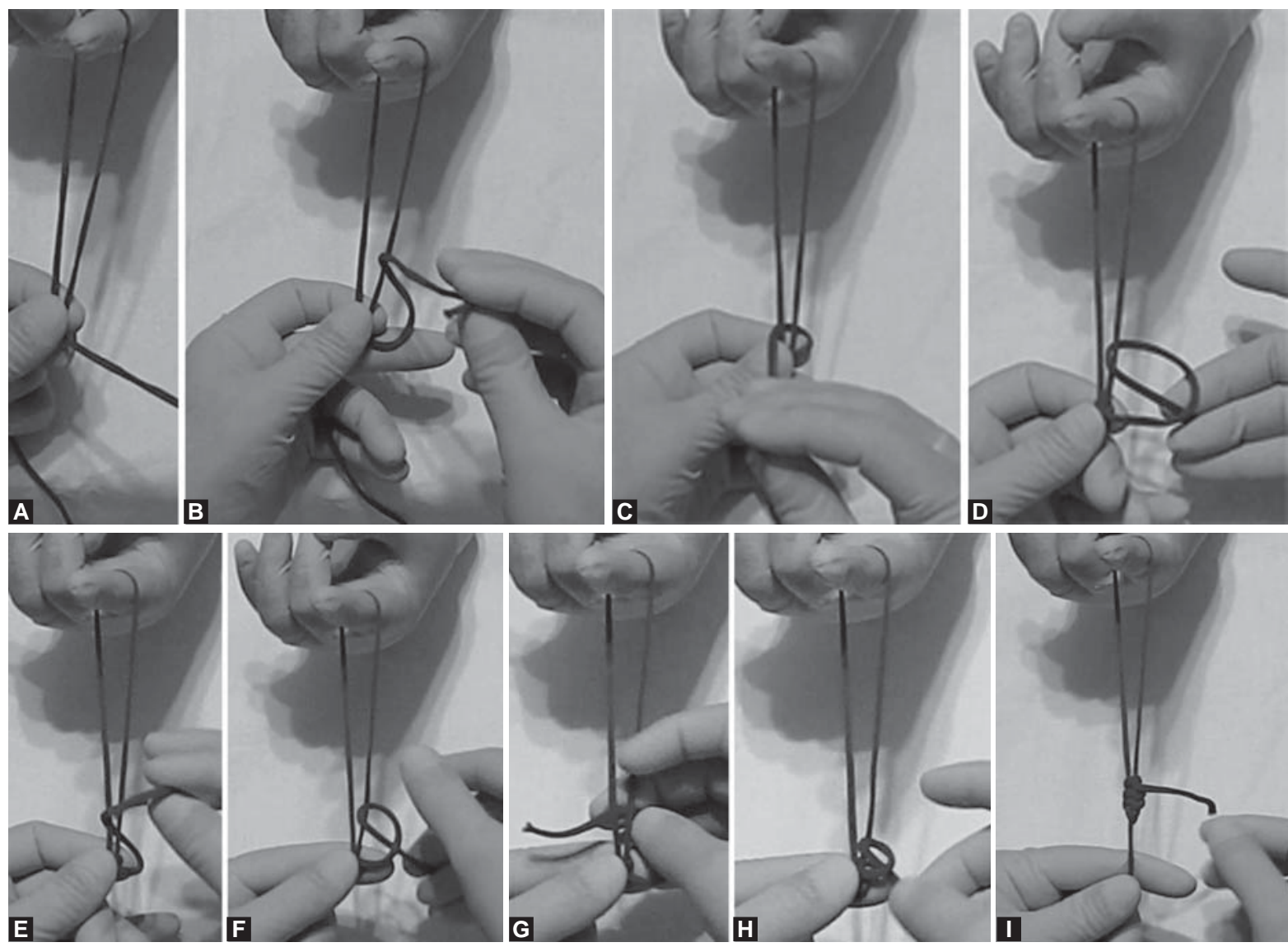

Figs 1A to I: Steps in tying Mishrals knot: (A) Place the short limb of the suture over the long limb, (B) take the first hinge,

$(C)$ take a wind, (D) make a half knot, (E) make the 2nd wind, $(F)$ again make the 2nd half knot, $(G)$ then make 3rd wind,

$\mathrm{(H)}$ and make the $3 \mathrm{rd}$ and final half knot and $(\mathrm{I})$ the final configuration of Mishrals knot
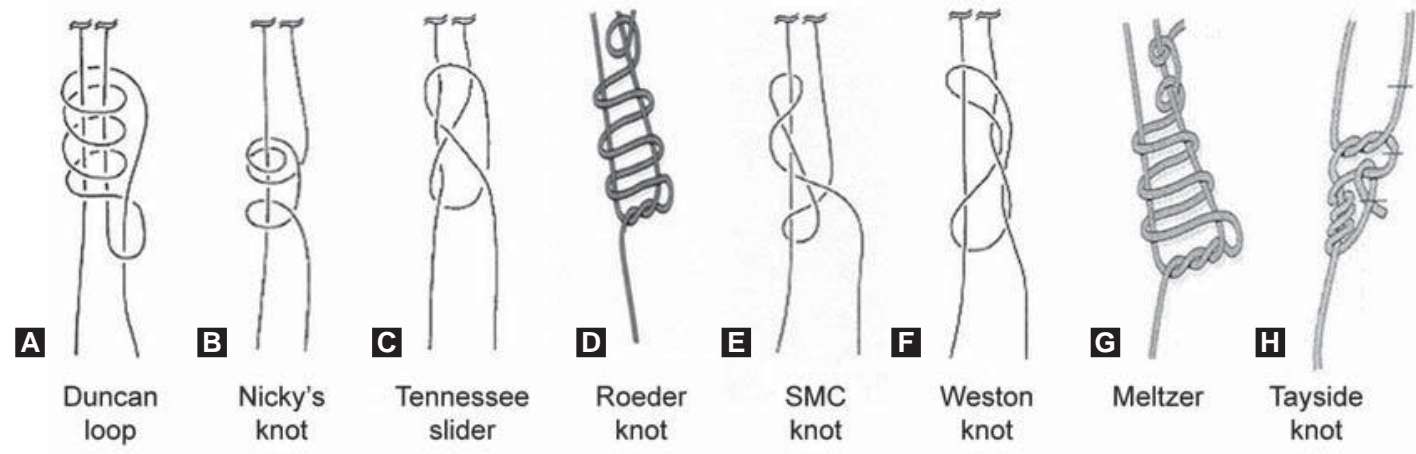

Figs 2A to H: Other common extracorporeal knots

difference was found in the other knot configurations when tied with no. 2 fiberwire.

Which knots provide the best balance of knot security and loop security? When evaluating all the knots, the knot that provided the best knot security and loop security in all cases, whether tying with no. 2 ethibond or no. 2 fiberwire, was the surgeon's knot. However, if one wishes to tie a sliding rather than a static knot, then the other knots must be considered (Table 2). When evaluating the sliding knots (Figs 2A to $\mathrm{H}$ ) without RHAPs tied with no. 2 ethibond, the Weston knot provided the best knot security, and the Duncan loop, Roeder knot, and Weston knot provided comparable loop security.
However, despite being the four best knots of the group, the Duncan loop, Mishra knot, Roeder knot, and Weston knot, had such poor loop security (all loop circumferences $32.5 \mathrm{~mm}$ ), that none of these knots are recommended to be tied without RHAPs. With either no. 2 fiberwire or no. 2 ethibond, the Roeder knot and Mishra knot tied with three RHAPs provide the best balance of loop and knot security of all the sliding knots tested.

\section{DISCUSSION}

Despite the great usefulness of laparoscopy for the treatment of surgical and gynecological diseases suture tying in the 
Table 2: Knot configurations providing optimal knot security and loop security in sliding knots tied with and without reversing halfhitches on alternating posts (RHAPs) using no. 2 ethibond or no. 2 fiberwire

\begin{tabular}{lllll}
\hline Knot type & Suture type & Best loop security & Best knot security & Recommendation \\
\hline Sliding knot (WR) & Ethibond & Duncan, Mishra, Roeder, Weston & Weston & None \\
Sliding knot (WR) & Fiberwire & Duncan & Weston & None \\
Sliding knot (R) & Ethibond & Roeder (R), Duncan (R), Nicky (R) & Weston (R), Roeder (R), & Roeder (R), Mishra (R) \\
& & & SMC (R), Mishra (R) & Mishra (R), Roeder (R) \\
Sliding knot (R) & Fiberwire & Roeder (R), Duncan (R), & Weston (R), Mishra (R) & Roeder (R) \\
& & Nicky (R), Mishra (R) & R) & \\
\end{tabular}

R: RHAPS; WR: Without RHAPS

cavity remains a great challenge. A knot to secure tissue approximation, which would be hand-made, secure, simple, easy, quick, reliable and extracorporeal without extra mechanical devices constitute the essence of surgical practice because an unreliable suture knot can spoil the outcomes of an otherwise beautifully performed surgical procedure. ${ }^{17}$ Optimization of both knot security and loop security ${ }^{3,11,22,23}$ for any given knot is critical, and recommendations regarding a specific knot should not be made without taking both characteristics under consideration.

Most of the studies showed that the loop security of almost all sliding knots tied without RHAPs was poor, hence RHAPs improve both the knot and loop securities. ${ }^{11}$ It is believed that this increased knot security occurs because the wrapping limbs tighten (removal of slack) around the post until the internal interference and friction are high enough to resist the applied load. ${ }^{20-22}$ Also, locking the knot by tensioning the wrapping limb and 'flipping' the knot also provided another potential mechanism of enlargement of the suture loop. ${ }^{12}$ Although this locking mechanism is particularly useful in preventing the knot from sliding back, locking the knot also causes expansion of the suture loop. ${ }^{7}$ This effect was seen in almost every knot that required a flipping maneuver to be locked. There has been previous classification of sliding knots as either lockable or nonlockable, with lockable knots further divided into proximal locking and distal-locking knot. ${ }^{5,13,16}$ In lockable sliding knots, tensioning the wrapping limb distorts the post limb, resulting in a kink in the post, thereby increasing the internal interference that increases the resistance of the knot from backing off. Clinically, after properly seating the knot at the repair site, the wrapping limb is tensioned, flipping the knot and preventing the knot from backing off. This locking effect is also known as the 'one-way ratchet effect' or the 'self-locking effect' ${ }^{\text {'16 }}$

Locking knots have previously been divided into proximal-locking and distal-locking knots (as referenced relative to the surgeon) according to where the wrapping limb deforms the post limb when it is tensioned. ${ }^{15,16}$ That is, a proximal-locking knot deforms in the portion of the knot that is closest to the surgeon, whereas a distal-locking knot deforms in the part of the knot that is furthest away from the surgeon. Proximal-locking knots include the Nicky's knot, and distal-locking knots include the Weston knot and Roeder knot. With the development of other knot configurations (the SMC knot), we propose that a third group be added, the middle-locking knot. In these knots, the wrapping limb emerges from the central part of the knot and include the SMC knot and the Tennessee slider. Mishra's knot appears to combines the characteristics of the three categories. ${ }^{7}$

\section{CONCLUSION}

A static surgeon's knot provides the best balance of loop security and knot security within the knot configurations tested. A sliding knot without RHAPs has both poor loop security and knot security and should not be tied. The addition of three RHAPs improves knot security of all sliding knots tested and improves loop security of most of the sliding knots tested. The addition of three RHAPs improved the knot security of all sliding knots to adequately resist predicted in vivo loads.

\section{REFERENCES}

1. Semm K. Tissue puncher and loop ligation, new ideas for surgical therapeutic pelvioscopy (laparoscopy) endoscopic intra-abdominal surgery. Endoscopy 1978;10:119-124.

2. Buess G, Mentges B, Manncke K, Starlinger M, Becker HD. Technique of transanal endoscopic microsurgery. Surg Endoscopy 1988;2:71-75

3. Burkhart SS, Wirth MA, Simonich M, et al. Knot security in simple sliding knots and its relationship to rotator cuff repair: how secure must the knot be? Arthroscopy 2000;16:202-207.

4. Budworth G. The book of practical fishing knots. Mechanicsburg, PA: Stackpole Books; 2003.

5. Burkhart SS, Wirth MA, Simonich M, et al. Loop security as a determinant of tissue fixation security. Arthroscopy 1998;14: 773-776.

6. Muffly TM, Kow N, Iqbal I, Barber MD. Minimum number of throws needed for knot security. J Surg Educ 2011;68(2): 130-133.

7. Mishra RK. Textbook of practical laparoscopic surgery. Jaypee Brothers Medical Publisher, New Delhi 2007; p.104-123.

8. Stephen W, Eubanks ES, Lee L, Swanstrom MD, Soper NJ, Lippincott Williams, Wilkins editors. 2004, 2nd ed. Mastery of endoscopic and laparoscopic surgery. 
9. Journal of Indian Association of Pediatric Surgeons 2010 OctDec:122-126. Available at: http://www.jiaps.com

10. Westebring-van der Putten EP, Goossens RHM, Jakimowicz JJ, Dankelman J. Haptics in minimally invasive surgery: a review. Minimally Invasive Therapy 2008;17(1):3-16.

11. Walid MS, Heaton RL. Laparoscopy-to-laparotomy quotient in obstetrics and gynecology residency programs. Arch Gyn Obs 2010;283(5):1027-1031.

12. Burkhart SS, Wirth MA, Simonich M, et al. Knot security in simple sliding knots and its relationship to rotator cuff repair: how secure must the knot be? Arthroscopy 2000;16:202-207.

13. Hage JJ. How capsizing, flipping and flyping of traditional knots can result in new endoscopic knots: a geometric review. J Am Coll Surg 2007;205(5):717-723.

14. Nottage WM, Lieurance RK. Current concepts: arthroscopic knot tying. Arthroscopy 1999;15:515-521.

15. Weston PV. A new clinch knot. Obstet Gynecol 1991;78:144-147.

16. Kim SH, Ha KI. The SMC knot: a new slip knot with locking mechanism. Arthroscopy 2000;16:563-565.
17. Chan KC, Burkhart SS. Arthroscopic knot tying. In: McGinty J, Burkhart S, Jackson R, et al, editors. Operative arthroscopy. Philadelphia: Lippincott, Williams and Wilkins, 2004; In press.

18. Lieurance RK, Pflaster DS, Abbott D, Nottage WM. Failure characteristics of various arthroscopically tied knots. Clin Orthop Relat Res 2003;408:311-318.

19. Croce E, Olmi S. Intracorporeal knot-tying and suturing techniques in laparoscopic surgery: technical details. JSLS 2000;4:17-22.

20. Ng JWT, Yeung B. Simple, instrument-assisted technique for tying a slip knot: a note of caution. ANZJ Surg 2004;74: 270-271.

21. Ilahi OA, Younas SA, Alexander J, Noble PC. Cyclic testing of arthroscopic knot security. Arthroscopy 2004;20:62-68.

22. Kriplani A, Bhatia P, Prasad A, Govil D, Garg HP. Comprehensive laparoscopic Surgery. Sagar, India 2007; p. 40-51.

23. Shettko DL, Frisbie DD, Hendrickson DA. A comparison of knot security of commonly used hand-tied laparoscopic slipknots. Vet Surg 2004;33(5):521-524. 